\title{
Balancing using accelerometers and equations with advanced arguments ${ }^{\star}$
}

\author{
Tamas Insperger * Richard Wohlfart* Janos Turi ** \\ Gabor Stepan* \\ * Budapest University of Technology and Economics, Department of \\ Applied Mechanics, Budapest, Hungary \\ (e-mail:inspi@mm.bme.hu,wohl@mm.bme.hu,stepan@mm.bme.hu) \\ ** Programs in Mathematical Sciences, University of Texas at Dallas, \\ Richardson, TX 75083 (e-mail: turi@utdallas.edu)
}

\begin{abstract}
The stick balancing problem is considered, where the vertical direction is measured using a single accelerometer attached to the stick. It is shown that the output is a linear combination of the angular position and the angular acceleration of the stick. If this output is fed back in a PD controller with feedback delay, then the governing equation of motion is an advanced functional differential equation, since the highest derivative, the jerk, appears with delayed argument through the derivative term. Autonomous equations with advanced arguments are typically non-causal and are unstable with infinitely many unstable poles. However, if the sampling effect of the digital controller is modeled, then the argument of the delayed highest derivative term is piecewise constant. In this case, the non-causality does not arise, and the system can also be stabilized by tuning the control parameters properly. In the paper, different models for stick balancing are considered and discussed by analyzing the corresponding stability diagrams.
\end{abstract}

Keywords: Feedback delay, Sampled data, Accelerometers, Advanced functional differential equations, Stability domains

\section{INTRODUCTION}

Systems, where the rate of change of the state depends on the state at deviating arguments are described by functional differential equations (FDEs). FDEs can be categorized into retarded, neutral and advanced types (see, e.g., Èl'sgol'c, 1964, or Kolmanovskii and Myshkis, 1999). If the rate of change of the state depends on the past states of the system, then the corresponding mathematical model is a retarded functional differential equation (RFDE). If the rate of change of the state depends on its own past values as well, then the corresponding equation is called neutral functional differential equation (NFDE). If the rate of change of the state depends on the past values of higher derivatives of the state, then the system is described by an advanced functional differential equation (AFDE). Note that these equations are also referred to as FDEs of retarded, neutral or advanced type (Kolmanovskii and Nosov, 1986).

The reason for the phrase "advanced" can be demonstrated by the following example. Consider the simple AFDE

\footnotetext{
^ This work was partially supported by the János Bolyai Research Scholarship of the Hungarian Academy of Sciences and the Hungarian National Science Foundation under grant no. K068910 and K72911 and by the National Science Foundation under grant DMS0705247 .
}

$$
\dot{x}(t)=\ddot{x}(t-\tau) .
$$

By a $\tau$-shift transformation in time, and by using the new variable $z=\dot{x}$, this equation can be written in the form

$$
\dot{z}(t)=z(t+\tau) .
$$

Here, the rate of change of state is determined by the future values of the state.

As opposed to RFDEs and NFDEs, AFDEs are rarely used in practical applications due to their inverted causality explained by (2). While linear autonomous RFDEs have infinitely many poles on the left half of the complex plane, linear autonomous AFDEs have infinitely many poles on the right half of the complex plane (Hale and Lunel, 1993, Niculescu, 2001). In this sense, linear autonomous AFDEs are always strongly or infinitely unstable.

Control systems with feedback delay are usually described by RFDEs or NFDEs. For instance, position and velocity feedback in a second order system results in an RFDE, while acceleration feedback induces an NFDE (see Vyhlídal et al., 2009). However, if the jerk is fed back in a second order system with feedback delay, then the governing equation is an AFDE, which is always unstable independently on the system and the control parameters. Although, jerk is practically never intended to be fed back in a control system, it may still appear in the input signal due to measuring error or noise. 


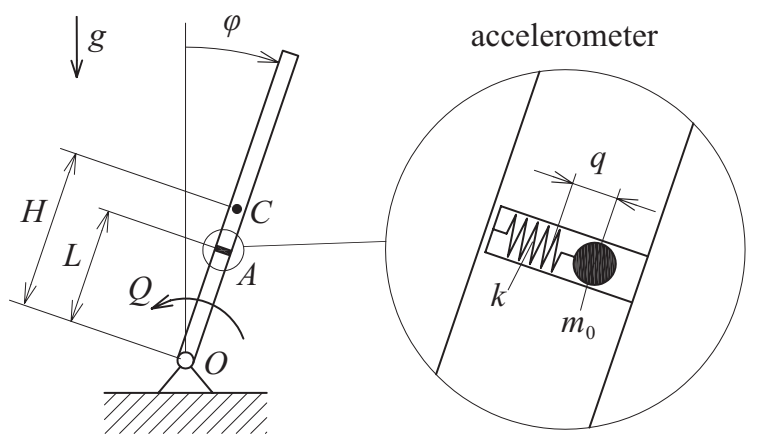

Fig. 1. One-DoF model of stick balancing, and the mechanical model of the accelerometer.

In this paper, a balancing problem is considered where the vertical direction is measured by accelerometers such that the jerk appears in the output. Different types of models are considered with respect to the arguments of the feedback term. The corresponding model equations are RFDEs, NFDEs, and AFDEs. It is shown that the sampling effect stabilizes the system even if an advanced term (the jerk with feedback delay) appears in the equation.

\section{DIFFERENT MECHANICAL MODELS}

Figure 1 presents the mechanical model of a simple stick balancing using the signal of an accelerometer to measure the angular position of the stick compared to the vertical direction. The equation of motion reads

$$
J \ddot{\varphi}(t)-H m g \sin \varphi(t)=Q(t),
$$

where $\varphi$ is the angular position of the pinned stick, $m$ is the mass, $H$ is the distance between the suspension point $O$ and the centre of gravity $C, J$ is the mass moment of inertia with respect to the axis normal to the plane of the figure through point $O$, and $Q$ is the control torque.

The angular position of the stick is measured by the piezo-accelerometer attached to the stick at point $A$. The mechanical model of the piezo-accelerometer is also presented in Fig. 1. This accelerometer operates normal to the stick as a mass $m_{0}$ attached to a spring of stiffness $k$ modeling the piezo crystal. The accelerometer's output $y$ is proportional to the displacement $q$ of the mass: $y=K_{A} q$ with $K_{A}[\mathrm{~V} / \mathrm{m}]$ being the characteristic constant of the accelerometer. The displacement $q$ of the mass depends on the angular position $\varphi$ of the rod. If the rod is standing still in an oblique position, then

$$
q=\frac{m_{0} g}{k} \sin \varphi
$$

Assuming small angular oscillations, the output can be approximated linearly as

$$
y=K \varphi,
$$

where $K=K_{A} m_{0} g / k[\mathrm{~V} / \mathrm{rad}]$.

If the dynamic effects, namely, the angular acceleration of the rod, are also taken into account, then the displacement of the accelerometer reads

$$
q=\frac{m_{0} g}{k} \sin \varphi-\frac{m_{0} L}{k} \ddot{\varphi} .
$$

In this case, the linearized output is

$$
y=K \varphi-K_{1} \ddot{\varphi},
$$

where $K_{1}=K_{A} m_{0} L / k\left[\mathrm{Vs}^{2} / \mathrm{rad}\right]$ and $K$ is the same as above. As it can be seen, the second derivative of the angular position appears in the output.

Based on the measurement technique of the output, three different models are distinguished:

(a) real-time continuous measurement: $y(t)$;

(b) continuous measurement with feedback delay: $y(t-\tau)$;

(c) sampled measurement with feedback delay (digital control): $y\left(t_{j-r}\right)$ with $t \in\left[t_{j}, t_{j+1}\right], t_{j}=j h, j \in \mathbb{Z}$, here $h$ is the sampling period, $r$ is the delay parameter and $\tau=r h$ is the feedback delay.

It is assumed that the output is fed back in a PD controller. In the case of sampled systems (digital controller), two derivative models can be defined:

(I) continuous differentiation: $\dot{y}\left(t_{j-r}\right), t \in\left[t_{j}, t_{j+1}\right]$;

(II) digital differentiation: $\frac{y\left(t_{j-r}\right)-y\left(t_{j-r-1}\right)}{h}, t \in\left[t_{j}, t_{j+1}\right]$.

Equations (5) and (7) and cases (a), (b), (c) and (I), (II) rises up 8 different models listed in table 1 . In the next section, these models are analyzed in details.

\section{ANALYSIS OF THE DIFFERENT MODELS}

In this section, the models listed in table 1 are considered and their stability properties are described briefly.

\section{Model 1.0}

In this model, it is assumed that the exact angular position is measured continuously real-time and is fed back without any delay. The corresponding linearized equation of motion reads

$$
\ddot{\varphi}(t)-\frac{H m g}{J} \varphi(t)=-\frac{P K}{J} \varphi(t)-\frac{D K}{J} \dot{\varphi}(t),
$$

which implies

$$
\ddot{\varphi}(t)+d \dot{\varphi}(t)+(p-a) \varphi(t)=0,
$$

with

$$
a=\frac{H m g}{J}, \quad p=\frac{P K}{J}, \quad d=\frac{D K}{J} .
$$

This system is asymptotically stable if $d>0$ and $p>a$.

\section{Model 1.1}

Here, it is assumed that the feedback loop contains a delay $\tau$. The corresponding linearized equation of motion reads

$$
\ddot{\varphi}(t)-a \varphi(t)=-p \varphi(t-\tau)-d \dot{\varphi}(t-\tau),
$$

where $a, p$ and $d$ are defined in (10). This equation is a basic equation for balancing with feedback delay (Stepan, 2009). Although complete pole placement is not possible for this system (Michiels, et al., 2002), it can be stabilized for certain system and delay parameters. The corresponding stability boundaries are the line $p=a$, and the parametric curve

$$
p=\left(\omega^{2}+a\right) \cos (\omega \tau), \quad d=\frac{\omega^{2}+a}{\omega} \sin (\omega \tau),
$$

with $\omega \in \mathbb{R}^{+}$. The corresponding stability diagram can be seen in Fig. 2 for $a=0.2$ and $\tau=1$. Note that this system is always unstable if $a>2$ (see, for instance, Stepan, 1989). 
Table 1. Different models for stick balancing using accelerometer

\begin{tabular}{cll}
\hline Name & output & control torque \\
\hline Model 1.0 & $y(t)=K \varphi(t)$ & $Q(t)=-P K \varphi(t)-D K \dot{\varphi}(t)$ \\
Model 1.1 & $y(t)=K \varphi(t-\tau)$ & $Q(t)=-P K \varphi(t-\tau)-D K \dot{\varphi}(t-\tau)$ \\
Model 1.2 & $y(t)=K \varphi\left(t_{j-r}\right)$ & $Q(t)=-P K \varphi\left(t_{j-r}\right)-D K \dot{\varphi}\left(t_{j-r}\right)$ \\
Model 1.3 & $y(t)=K \varphi\left(t_{j-r}\right)$ & $Q(t)=-P K \varphi\left(t_{j-r}\right)-D K \frac{\varphi\left(t_{j-r}\right)-\varphi\left(t_{j-r-1}\right)}{h}$ \\
Model 2.0 & $y(t)=K \varphi(t)-K_{1} \ddot{\varphi}(t)$ & $Q(t)=-P K \varphi(t)+P K_{1} \ddot{\varphi}(t)-D K \dot{\varphi}(t)+D K_{1} \dddot{\varphi}(t)$ \\
Model 2.1 & $y(t)=K \varphi(t-\tau)-K_{1} \ddot{\varphi}(t-\tau)$ & $Q(t)=-P K \varphi(t-\tau)+P K_{1} \ddot{\varphi}(t-\tau)-D K \dot{\varphi}(t-\tau)+D K_{1} \dddot{\varphi}(t-\tau)$ \\
Model 2.2 & $y(t)=K \varphi\left(t_{j-r}\right)-K_{1} \ddot{\varphi}\left(t_{j-r}\right)$ & $Q(t)=-P K \varphi\left(t_{j-r}\right)+P K_{1} \ddot{\varphi}\left(t_{j-r}\right)-D K \dot{\varphi}\left(t_{j-r}\right)+D K_{1} \dddot{\varphi}\left(t_{j-r}\right)$ \\
Model 2.3 & $y(t)=K \varphi\left(t_{j-r}\right)-K_{1} \ddot{\varphi}\left(t_{j-r}\right)$ & $Q(t)=-P K \varphi\left(t_{j-r}\right)+P K_{1} \ddot{\varphi}\left(t_{j-r}\right)-D K \frac{\varphi\left(t_{j-r}\right)-\varphi\left(t_{j-r-1}\right)}{h}+D K_{1} \frac{\ddot{\varphi}\left(t_{j-r}\right)-\ddot{\varphi}\left(t_{j-r-1}\right)}{h}$ \\
\hline
\end{tabular}
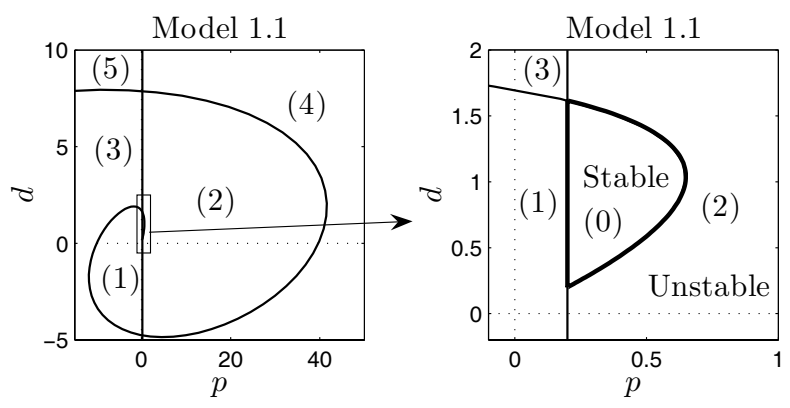

Fig. 2. Stability boundaries and the number of unstable poles for (11) with $a=0.2$.

\section{Model 1.2}

In this model, it is assumed that sampling effect (or zero order hold) also arises in addition to the feedback delay. The governing equation reads

$$
\ddot{\varphi}(t)-a \varphi(t)=-p \varphi\left(t_{j-r}\right)-d \dot{\varphi}\left(t_{j-r}\right), \quad t \in\left[t_{j}, t_{j+1}\right],
$$

where $t_{j}=j h, j \in \mathbb{Z}$ denotes the discrete instants of sampling, $h$ is the sampling period and $r$ is the delay parameter. The feedback delay in this case is $\tau=r h$. The parameters $a, p$ and $d$ are defined in (10).

Note that this equation can be represented also in the form

$$
\ddot{\varphi}(t)-a \varphi(t)=-p \varphi(t-\sigma(t))-d \dot{\varphi}(t-\sigma(t)),
$$

where

$$
\sigma(t)=\tau-t_{j}+t, \quad t \in\left[t_{j}, t_{j+1}\right)
$$

is a time-periodic time delay shown in Fig. 3. In fact, the sampling effect introduces a periodic parametric excitation at the time delay according to (15), thus (13) is an nonautonomous RFDE. It can be seen that the time-periodic time delay $\sigma(t)$ tends to the constant delay $\tau$ if the sampling period $h$ tends to 0 and $r$ tends to $\infty$ by keeping $r h=\tau$. This limit is basically the key point of the semidiscetization method of delayed system (see, Insperger et al., 2008).

Equation (13) can be transformed to the form

$$
\begin{aligned}
\dot{x}(t) & =A x(t)+B u\left(t_{j-r}\right), \quad t \in\left[t_{j}, t_{j+1}\right] \\
u\left(t_{j}\right) & =D x\left(t_{j}\right)
\end{aligned}
$$
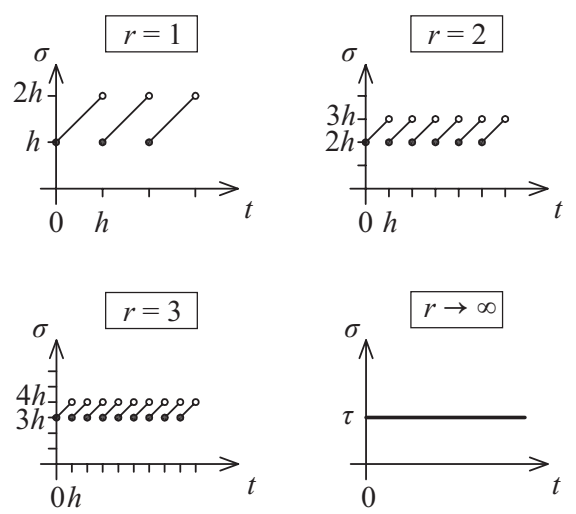

Fig. 3. Sampling effect as time-periodic delay.

$$
x(t)=\left(\begin{array}{c}
\varphi(t) \\
\dot{\varphi}(t)
\end{array}\right), A=\left(\begin{array}{ll}
0 & 1 \\
a & 0
\end{array}\right), B=\left(\begin{array}{l}
0 \\
1
\end{array}\right), D=(-p-d) .
$$

Piecewise solution of the system over a sampling period and state augmentation gives the finite dimensional discrete map

$$
\left(\begin{array}{c}
x_{j+1} \\
u_{j} \\
u_{j-1} \\
\vdots \\
u_{j-r+1}
\end{array}\right)=\underbrace{\left(\begin{array}{ccccc}
P & 0 & \ldots & 0 & R B \\
D & 0 & \ldots & 0 & 0 \\
0 & 1 & \ldots & 0 & 0 \\
\vdots & \ddots & & \vdots \\
0 & 0 & \ldots & 1 & 0
\end{array}\right)}_{=\Phi_{1.2}}\left(\begin{array}{c}
x_{j} \\
u_{j-1} \\
u_{j-2} \\
\vdots \\
u_{j-r}
\end{array}\right)
$$

where $x_{j}=x\left(t_{j}\right), u_{j}=u\left(t_{j}\right)$, and

$$
P=\mathrm{e}^{A h}, \quad R=\int_{0}^{h} \mathrm{e}^{A(h-s)} \mathrm{d} s .
$$

In the case $r=0$ (when only zero order hold appears in the feedback loop without any delay), the discrete map turns to the form

$$
x_{j+1}=\underbrace{(P+R B D)}_{=\Phi_{1.2}} x_{j} .
$$

The system is asymptotically stable if the eigenvalues of the monodromy matrix $\Phi_{1.2}$ in (19) or in (21) are in modulus less than one. The corresponding stability diagram can be seen in Fig. 4 for $a=0.2$.

If the delay $\tau=r h$ is fixed to 1 , then the system is always unstable if

with 


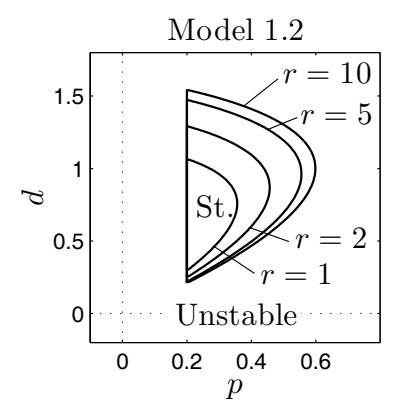

Fig. 4. Stability boundaries for (13) for different delay parameters $r$ with $r h=\tau=1$ and $a=0.2$.

$$
a>a_{\text {crit }}=\left(r \ln \left(\frac{r(r+1)+1+\sqrt{2 r(r+1)+1}}{r(r+1)}\right)\right)^{2} .
$$

For $r=1$, this formula reduces to

$$
a_{\text {crit }}=\ln ^{2}\left(\frac{3+\sqrt{5}}{2}\right)=0.926
$$

that was derived for the digitally controlled inverted pendulum in Enikov and Stepan (1998). The critical values to different delay parameters $r$ are summarized in table 2. It can be seen that as $r \rightarrow \infty$ and $h \rightarrow 0$ by keeping $\tau=r h=1$ constant, the critical system parameter tends to that of Model 1.1: $a_{\text {crit }}=2$.

Table 2. Some critical system parameters for Model 1.2 with $\tau=r h=1$

\begin{tabular}{cccccc}
\hline$r$ & 1 & 2 & 5 & 10 & 100 \\
\hline$a_{\text {crit }}$ & 0.926 & 1.297 & 1.658 & 1.815 & 1.980 \\
\hline
\end{tabular}

\section{Model 1.3}

The new feature of this model compared to the previous one is that here the angular velocity for the derivative term of the feedback is determined by digital differentiation. The corresponding equation reads

$$
\ddot{\varphi}(t)-a \varphi(t)=-p \varphi\left(t_{j-r}\right)-d \frac{\varphi\left(t_{j-r}\right)-\varphi\left(t_{j-r-1}\right)}{h},
$$

This system can be transformed to a system with two delays

$$
\ddot{\varphi}(t)-a \varphi(t)=-p_{1} \varphi\left(t_{j-r}\right)+p_{2} \varphi\left(t_{j-r-1}\right), \quad t \in\left[t_{j}, t_{j+1}\right],
$$

where $p_{1}=p+d / h$ and $p_{2}=d / h$. The corresponding state-space model reads

$$
\begin{aligned}
\dot{x}(t) & =A x(t)+B D_{1} y\left(t_{j-r}\right)+B D_{2} y\left(t_{j-r-1}\right), \\
y\left(t_{j}\right) & =C x\left(t_{j}\right), \quad t \in\left[t_{j}, t_{j+1}\right]
\end{aligned}
$$

with $x(t), A$, and $B$ defined in (18) and

$$
C=\left(\begin{array}{ll}
1 & 0
\end{array}\right), \quad D_{1}=\left(-p_{1}\right), \quad D_{2}=\left(p_{2}\right) .
$$

This semi-discrete system implies the discrete map

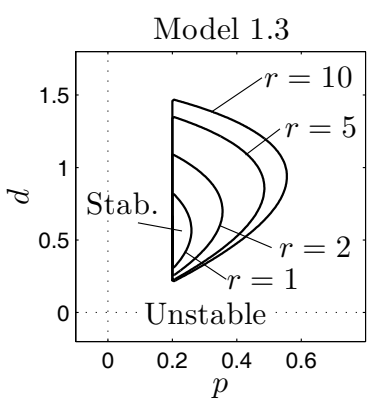

Fig. 5. Stability boundaries for (24) for different delay parameters $r$ with $r h=\tau=1$ and $a=0.2$.

$$
\left(\begin{array}{c}
x_{j+1} \\
y_{j} \\
y_{j-1} \\
\vdots \\
y_{j-r}
\end{array}\right)=\underbrace{\left(\begin{array}{ccccccc}
P & 0 & \ldots & 0 & R B D_{1} & R B D_{2} \\
C & 0 & \ldots & 0 & 0 & 0 \\
0 & 1 & \ldots & 0 & 0 & 0 \\
\vdots & \ddots & & \vdots \\
0 & 0 & \ldots & 1 & 0
\end{array}\right)}_{=\Phi_{1.3}}\left(\begin{array}{c}
x_{j} \\
y_{j-1} \\
y_{j-2} \\
\vdots \\
y_{j-r-1}
\end{array}\right)
$$

where $P$ and $R$ are defined in (20). If $r=0$, the discrete map has the form

$$
\left(\begin{array}{c}
x_{j+1} \\
y_{j}
\end{array}\right)=\underbrace{\left(\begin{array}{cc}
P+R B D_{1} C & R B D_{2} \\
C & 0
\end{array}\right)}_{=\Phi_{1.3}}\left(\begin{array}{c}
x_{j} \\
y_{j-1}
\end{array}\right) .
$$

The system is asymptotically stable if the eigenvalues of the monodromy matrix $\Phi_{1.3}$ in (29) or in (30) are in modulus less than one. The associated stability diagram is presented in Fig. 5 for $a=0.2$.

Model 2.0

In this model, it is assumed that the angular position is measured by accelerometers continuously without any feedback delay. In this case the output of the system is affected by the angular acceleration of the stick, and the equation of motion reads

$$
\begin{aligned}
\ddot{\varphi}(t)-\frac{H m g}{J} \varphi(t)=-\frac{P K}{J} \varphi(t)+\frac{P K_{1}}{J} \ddot{\varphi}(t) \\
\quad-\frac{D K}{J} \dot{\varphi}(t)+\frac{D K_{1}}{J} \dddot{\varphi}(t) .
\end{aligned}
$$

This equation can be written in the form

$$
-\varepsilon d \dddot{\varphi}(t)+(1-\varepsilon p) \ddot{\varphi}(t)+d \dot{\varphi}(t)+(p-a) \varphi(t)=0,
$$

where $\varepsilon=K_{1} / K$ describes the weight of the angular acceleration in the output, and the parameters $a, p$ and $d$ are defined in (10). The condition for asymptotic stability for this ordinary differential equation is

$$
d>0 \text { and } a<p<1 / \varepsilon<0 .
$$

Clearly, this system is unstable for any parameters $p$ and $d$, if $a>0$.

\section{Model 2.1}

This model assumes that the output of the system is affected by the angular acceleration as in the previous model, but the feedback loop involves a delay $\tau$. The corresponding equation of motion reads 
$\ddot{\varphi}(t)-a \varphi(t)=-p \varphi(t-\tau)+\varepsilon p \ddot{\varphi}(t-\tau)-d \dot{\varphi}(t-\tau)+\varepsilon d \dddot{\varphi}(t-\tau)$

where, again, $a, p$ and $d$ are defined in (10), and $\varepsilon=K_{1} / K$. As it can be seen, the highest derivative appears with a delayed argument, thus this equation is an autonomous AFDE. Consequently, this system is always unstable with infinitely many unstable poles if $\varepsilon d \neq 0$.

\section{Model 2.2}

Here, the sampling effect is also modeled, and the governing equation is shaped as

$$
\begin{aligned}
& \ddot{\varphi}(t)-a \varphi(t)=-p \varphi\left(t_{j-r}\right)+\varepsilon p \ddot{\varphi}\left(t_{j-r}\right) \\
& \quad-d \dot{\varphi}\left(t_{j-r}\right)+\varepsilon d \dddot{\varphi}\left(t_{j-r}\right), \quad t \in\left[t_{j}, t_{j+1}\right] .
\end{aligned}
$$

This equation can also be considered as an AFDE, since the highest derivative appears with a delayed argument. Note that the term $\dddot{\varphi}\left(t_{j-r}\right)$ with $t \in\left[t_{j}, t_{j+1}\right]$ can be written in the form $\dddot{\varphi}(t-\sigma(t))$ with $\sigma(t)$ defined in (15) (see also Fig. 3). Still, this equation does not face to the non-causality of (34), since this equation can simply be solved by the method of steps, if the initial function and its appropriate derivatives are given. The corresponding state-space model reads

$$
\begin{aligned}
\dot{x}(t) & =A x(t)+B u\left(t_{j-r}\right), \quad t \in\left[t_{j}, t_{j+1}\right] \\
u\left(t_{j}\right) & =D x\left(t_{j}\right)-\varepsilon D \ddot{x}\left(t_{j}\right)
\end{aligned}
$$

with $x(t), A, B$, and $D$ defined in (18). Piecewise solution of the system over a sampling period and state augmentation gives the finite dimensional discrete map

$$
\left(\begin{array}{c}
x_{j+1} \\
\ddot{x}_{j+1} \\
u_{j} \\
u_{j-1} \\
\vdots \\
u_{j-r+1}
\end{array}\right)=\underbrace{\left(\begin{array}{ccccc}
P & 0 & \ldots & 0 & R B \\
A^{2} P & 0 & \ldots & 0 & Q B \\
D & -\varepsilon D & & 0 & 0 \\
0 & 1 & & 0 & 0 \\
\vdots & & \ddots & & \vdots \\
0 & 0 & \ldots & 1 & 0
\end{array}\right)}_{=\Phi_{2.2}}\left(\begin{array}{c}
x_{j} \\
\ddot{x}_{j} \\
u_{j-1} \\
u_{j-2} \\
\vdots \\
u_{j-r}
\end{array}\right),
$$

where $P$ and $R$ are the same as in (20), and

$$
Q=\int_{0}^{h} A^{2} \mathrm{e}^{A(h-s)} \mathrm{d} s+A .
$$

If $r=0$ then the discrete map turns to the form

$$
\left(\begin{array}{c}
x_{j+1} \\
\ddot{x}_{j}
\end{array}\right)=\underbrace{\left(\begin{array}{c}
P+R B D \\
A^{2} P+Q R B D \\
A^{2}+\varepsilon B D
\end{array}\right)}_{=\Phi_{2.2}}\left(\begin{array}{c}
x_{j} \\
\ddot{x}_{j-1}
\end{array}\right) .
$$

The system is asymptotically stable if the eigenvalues of the monodromy matrix $\Phi_{2.2}$ in (38) or (40) in are in modulus less than one. The stability boundaries can be seen in Fig. 6 for $a=0.2$ with different $r$ and $\varepsilon$ values. It can be seen that there exist some domains of the control parameters, where the system is stable. Note that the case $\varepsilon=0$ gives Model 1.2, and the corresponding stability boundaries are identical to that of Model 1.2.

\section{Model 2.3}

In this model, the angular velocity for the derivative term of the feedback is determined by digital differentiation as it was done for Model 1.3. The corresponding equation reads
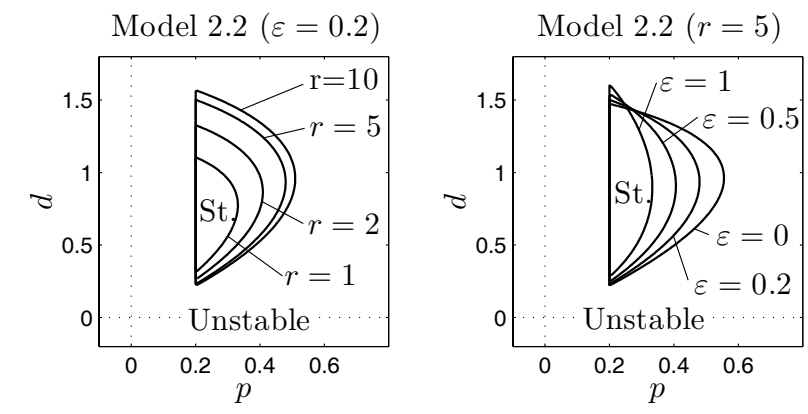

Fig. 6. Stability boundaries for (35) for different delay parameters $r$ (left) and for different $\varepsilon$ (right) with $r h=\tau=1$ and $a=0.2$.

$$
\begin{aligned}
& \ddot{\varphi}(t)-a \varphi(t)=-p \varphi\left(t_{j-r}\right)+\varepsilon p \ddot{\varphi}\left(t_{j-r}\right) \\
& -d \frac{\varphi\left(t_{j-r}\right)-\varphi\left(t_{j-r-1}\right)}{h}+\varepsilon d \frac{\ddot{\varphi}\left(t_{j-r}\right)-\ddot{\varphi}\left(t_{j-r-1}\right)}{h} \\
& t \in\left[t_{j}, t_{j+1}\right] .
\end{aligned}
$$

In this case, the highest derivative appears both with delayed and with non-delayed arguments, thus, this equation is an NFDE. Similarly to (41), this system can be transformed to a system with two delays

$$
\begin{aligned}
\ddot{\varphi}(t) & -a \varphi(t)=-p_{1} \varphi\left(t_{j-r}\right)+p_{2} \varphi\left(t_{j-r-1}\right) \\
& +\varepsilon p_{1} \ddot{\varphi}\left(t_{j-r}\right)-\varepsilon p_{2} \ddot{\varphi}\left(t_{j-r-1}\right), \quad t \in\left[t_{j}, t_{j+1}\right],
\end{aligned}
$$

where $p_{1}=p+d / h$ and $p_{2}=d / h$. The corresponding state-space model reads

$$
\begin{array}{rlr}
\dot{x}(t) & =A x(t)+B D_{1} y\left(t_{j-r}\right)+B D_{2} y\left(t_{j-r-1}\right), \\
y\left(t_{j}\right) & =C_{1} x\left(t_{j}\right)+C_{2} \ddot{x}\left(t_{j}\right), & t \in\left[t_{j}, t_{j+1}\right],
\end{array}
$$

where $x(t), A$, and $B$ are defined in (18) and

$$
\begin{aligned}
C_{1} & =\left(\begin{array}{ll}
1 & 0
\end{array}\right), \quad C_{2}=\left(\begin{array}{l}
-\varepsilon \\
-\varepsilon
\end{array}\right), \\
D_{1} & =\left(\begin{array}{ll}
-p_{1}
\end{array}\right), \quad D_{2}=\left(-p_{2}\right) .
\end{aligned}
$$

The corresponding discrete map reads

$$
\left(\begin{array}{c}
x_{j+1} \\
\ddot{x}_{j+1} \\
y_{j} \\
y_{j-1} \\
\vdots \\
y_{j-r}
\end{array}\right)=\underbrace{\left(\begin{array}{ccccccc}
P & 0 & \ldots & 0 & R B D_{1} & R B D_{2} \\
A^{2} P & 0 & \ldots & 0 & Q B D_{1} & Q B D_{2} \\
C_{1} & C_{2} & \ldots & 0 & 0 & 0 \\
0 & 1 & \ldots & 0 & 0 & 0 \\
\vdots & & \ddots & & \vdots \\
0 & 0 & \ldots & 1 & 0
\end{array}\right)}_{=\Phi_{2.3}}\left(\begin{array}{c}
x_{j} \\
\ddot{x}_{j} \\
y_{j-1} \\
y_{j-2} \\
\vdots \\
y_{j-r-1}
\end{array}\right),
$$

where $P$ and $R$ are defined in (20) and $Q$ is defined in (39). If $r=0$, the discrete map has the form

$$
\left(\begin{array}{c}
x_{j+1} \\
\ddot{x}_{j+1} \\
y_{j}
\end{array}\right)=\underbrace{\left(\begin{array}{ccc}
P+R B D_{1} C_{1} & R B D_{1} C_{2} & R B D_{2} \\
A^{2} P+Q B D_{1} C_{1} & Q B D_{1} C_{2} & Q B D_{2} \\
C_{1} & 0
\end{array}\right)}_{=\Phi_{2.3}}\left(\begin{array}{c}
x_{j} \\
\ddot{x}_{j} \\
C_{j-1}
\end{array}\right) .
$$

The system is asymptotically stable if the eigenvalues of the monodromy matrix $\Phi_{2.3}$ in (47) or (48) are in modulus less than one. The corresponding stability diagram is presented in Fig. 7 for $a=0.2$ with different $r$ and $\varepsilon$. Note that the case $\varepsilon=0$ gives Model 1.3, and the corresponding stability boundaries are identical to that of Model 1.3. 

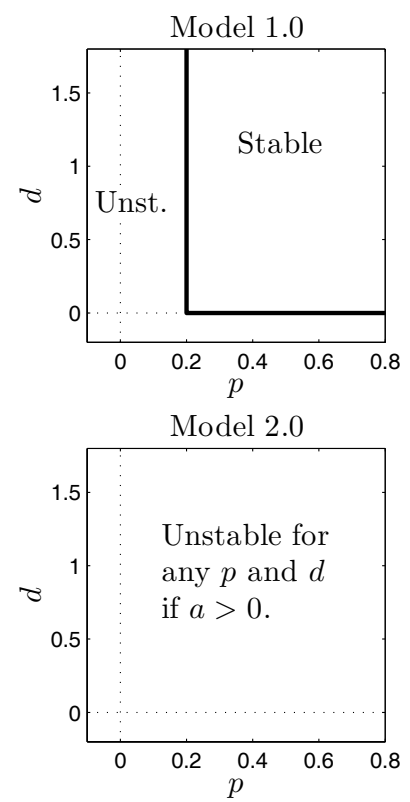

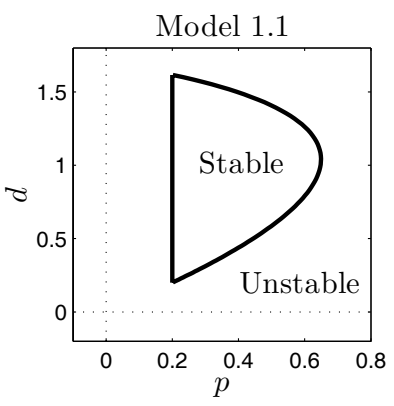

Model 2.1

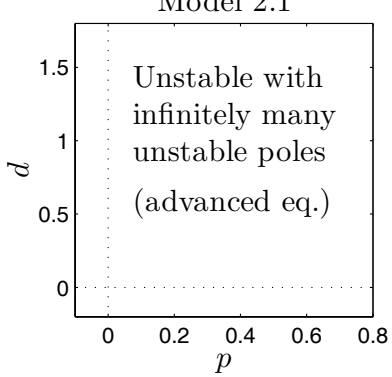

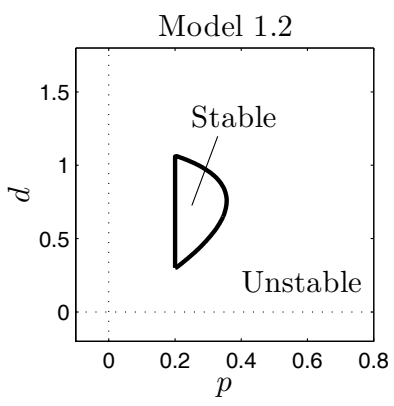

Model 2.2

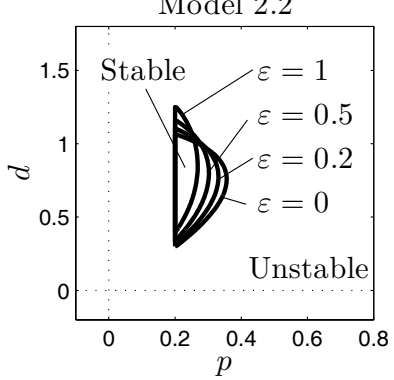

Model 1.3

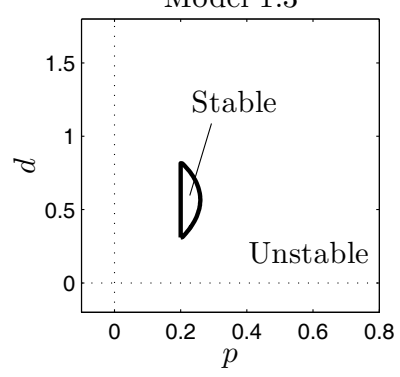

Model 2.3

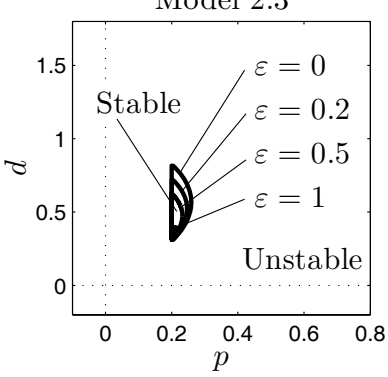

Fig. 8. Stability diagrams for the different models. Parameters: $a=0.2, \tau=1$ (for models 1.1 and 2.1 ) and $r=1$ (for models $1.2,1.3,2.2$ and 2.3$)$.
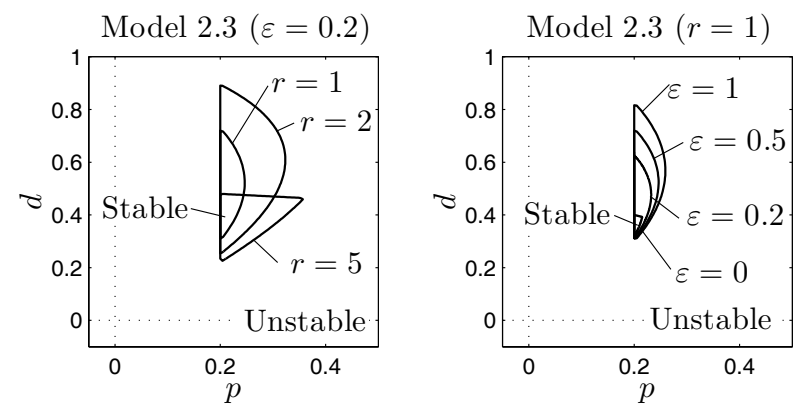

Fig. 7. Stability boundaries for (41) for different delay parameters $r$ (left) and for different $\varepsilon$ (right) with $r h=\tau=1$ and $a=0.2$.

\section{RESULTS}

Stability diagrams to the different models listed in table 1 are summarized in Fig. 8. The cases, when the signal of the accelerometers is fed back continuously without delay (Model 2.0) results in a system, that cannot be stabilized, if the open loop system is unstable (i.e., if $a<0$ ). If signal of the accelerometers is fed back continuously with feedback delay (Model 2.1), then the equation of motion is an AFDE, and the system is unstable with infinitely many unstable poles. This strong instability is however reversed if the sampling effect of a (digital) controller is also considered in the model. A detailed study about this phenomenon for first order delayed, neutral and advanced scalar equations were presented in Insperger et al. (2010).

\section{REFERENCES}

Èl'sgol'c, L.È. (1964). Qualitative Methods in Mathematical Analysis. American Mathematical Society.

Enikov, E., and Stepan, G. (1998). Micro-chaotic motion of digitally controlled machines. Journal of Vibration and Control, 4(4), pp. 427-443.
Hale, J.K. \& Lunel, S.M.V. (1993). Introduction to Functional Differential Equations. Springer-Verlag.

Insperger, T., Stepan, G. \& Turi, J. (2008). On the higherorder semi-discretizations for periodic delayed systems. Journal of Sound and Vibration 313, 334-341.

Insperger, T., Stepan, G. \& Turi, J. (2010) Delayed feedback of sampled higher derivatives, Philosophical Transactions of the Royal Society A, 368, 469-482.

Kolmanovskii, V.B. \& Myshkis, A.D. (1999). Introduction to the theory and applications of functional differential equations. Kluwer Academic Publishers.

Kolmanovskii, V.B. \& Nosov, V.R. (1986). Stability of Functional Differential equations. Academic Press.

Michiels, W., Engelborghs, K., Vansevenant, P. \& Roose, D. (2002). Continuous pole placement for delay equations. Automatica 38(5), 747-761.

Niculescu, S.-I. 2001 Delay effects on stability - A robust control approach, Springer-Verlag.

Stepan, G. (1989). Retarded Dynamical Systems, Longman.

Stepan, G. (2009). Delay effects in the human sensory system during balancing. Philosophical Transactions of The Royal Society A - Mathematical Physical and Engineering Sciences 367, 1195-1212.

Vyhlídal, T., Michiels, W., Zítek, P. \& McGahan, P. (2009). Stability impact of small delays in proportionalderivative state feedback. Control Engineering Practice 17, 382-393. 